\title{
Rg1 exhibits neuroprotective effects by inhibiting the endoplasmic reticulum stress-mediated c-Jun N-terminal protein kinase apoptotic pathway in a rat model of Alzheimer's disease
}

\author{
JUN-SHAN MU, HANG LIN, JIAN-XIN YE, MIN LIN and XIAO-PING CUI \\ Department of Neurology, Fuzhou General Hospital and Clinical Medical College of Fujian Medical University, \\ Fuzhou, Fujian 350025 P.R. China
}

Received March 13, 2014; Accepted December 17, 2014

DOI: $10.3892 / \mathrm{mmr} .2015 .3853$

\begin{abstract}
The neuroprotective agents currently used to treat Alzheimer's disease (AD) often only target one aspect of the disease process. Therefore, identifying effective drug targets associated with the pathogenesis of AD is critical for the production of novel AD therapeutic strategies. The present study aimed to investigate the underlying mechanisms of the neuroprotective effects of $\operatorname{Rg} 1$ on a rat model of $\mathrm{AD}$. A double transgenic $\beta$-amyloid (A $\beta$ ) precursor protein/PS1 rat model was established, which co-expressed mutations associated with $\mathrm{AD}$. A $\beta$ plaques and neurofibrillary tangles (NFTs) were detected by immunohistochemistry. The detection of the protein expression levels of caspase-3 and terminal deoxynucleotidyl-transferase-mediated dUTP nick end labeling (TUNEL) staining were used to determine the level of apoptosis in the brain tissue. The expression levels of the endoplasmic reticulum (ER) stress biomarker, glucose-regulated protein 78 (Grp78), and the mitochondrial apoptosis biomarkers, B-cell lymphoma 2 (Bcl-2) and Bcl-2-associated X protein (Bax), were analyzed by western blotting. Furthermore, the expression of the proteins associated with the ER stress unfolded protein response (UPR) was determined, in order to examine the levels of ER stress. The mRNA expression of downstream genes of UPR were also detected by reverse transcription-polymerase chain reaction. The protein expression levels of the apoptosis-associated phosphorylated-c-Jun $\mathrm{N}$-terminal protein kinase ( $\mathrm{p}-\mathrm{JNK}$ ), caspase-12 and cAMP response element-binding transcription factor homologous
\end{abstract}

Correspondence to: Dr Jun-Shan Mu, Department of Neurology, Fuzhou General Hospital and Clinical Medical College of Fujian Medical University, 156 Xi'erhuan North Road, Fuzhou, Fujian 350025, P.R. China

E-mail: mujunshandr@yeah.net

Key words: Alzheimer's disease, ginsenoside Rg1, endoplasmic reticulum stress, c-Jun $\mathrm{N}$-terminal protein kinase apoptotic pathway, tumor necrosis factor receptor-associated factor 2 protein were determined by western blotting. The results of the present study indicated that the accumulation of NFTs and $\mathrm{A} \beta$ plaques was significantly decreased in the Rg1-treated $\mathrm{AD}$ rats, compared with untreated $\mathrm{AD}$ rats. The expression of caspase-3 and the number of TUNEL-positive cells were also significantly decreased in the Rg1-treated rats, as compared with the AD rats. Furthermore, treatment with Rg1 significantly reduced the expression of Grp78, and triggered inositol-requiring enzyme-1 (IRE-1) and phosphorylated protein kinase RNA-like ER kinase-associated ER stress. The IRE-1 UPR pathway downstream gene, tumor necrosis factor receptor-associated factor 2 , was significantly decreased in rats treated with $\mathrm{Rg} 1$, compared with untreated $\mathrm{AD}$ rats. Furthermore, the activation of p-JNK was also inhibited when $\mathrm{AD}$ rats were treated with Rg1. In conclusion, Rg1 was shown to function as an important factor that inhibits the accumulation of NFTs and $A \beta$ via inhibition of the ER stress-mediated pathway. Blocking of this pathway was triggered by the IRE-1 and TRAF2 pathway, as a result of inhibition of the expression of $\mathrm{p}-\mathrm{JNK}$.

\section{Introduction}

Alzheimer's disease (AD) is a progressive neurodegenerative disorder of the central nervous system, which is characterized by a progressive loss of memory, deficits in cognitive function and dementia $(1,2)$. AD is neuropathologically characterized by extracellular senile plaques that contain $\beta$-amyloid $(A \beta)$ precursor protein (APP), and intracellular neurofibrillary tangles (NFTs), which occur as a result of hyperphosphorylation of the tau protein $(3,4)$. The currently available treatment methods suppress a number of the symptoms of AD, however they are unable to halt of reverse the progression of this disease (5).

Numerous neuroprotective drugs have been discovered in animal studies $(2,4)$. However, no drug has been shown to significantly slow or stop the progression of AD. This may be due to the multiple pathways involved in the pathogenesis of $\mathrm{AD}$, including mitochondrial dysfunction, short-term and long-term oxidative stress, energy crises, excitotoxicity, neuroinflammation and protein aggregation (6). Furthermore, the 
available neuroprotective agents often only target one aspect of the disease process. Therefore, identifying effective drug targets that are associated with the pathogenesis of AD is critical for the development of novel AD therapeutic strategies.

Ginsenoside $\mathrm{Rg} 1$ is a steroidal saponin that is highly abundant in ginseng, in which ginsenoside $\operatorname{Rg} 1$ is one of the most important components (7). Recent studies have identified neuroprotective effects of $\operatorname{Rg} 1(8,9)$. However, the neuroprotective effects of Rg1 on an AD animal model, and the underlying mechanisms, have yet to be elucidated. Therefore, the aim of the present study was to examine the effects of ginsenoside Rg1 on an animal model of AD, and to determine the mechanisms underlying any such effects.

\section{Materials and methods}

Animals. Female Wistar rats ( $\sim 3$ months old; Animal Center of Fuzhou Medical University, Fuzhou, China), were used in the present study. The rats were housed in a temperature-controlled environment, with a $12 \mathrm{~h}$ light/dark cycle and ad libitum access to standard feed and water, which was limited to $4 \mathrm{~h}$ per day. All animal experimentation was conducted in accordance with the Canadian Council on Animal Care guidelines for the Care and Use of Laboratory Animals, and was approved by Fujian Medical University (Fuzhou, China). The present study was approved by the Ethics Committee of Fuzhou General Hospital and Clinical Medical College of Fujian Medical University.

Establishment of an AD model. A double transgenic APP/PS1 rat model (AD rats) was established, which co-expressed mutations associated with AD that are known to lead to accelerated plaque formation and increased $A \beta$ production. The model was generated by introducing the APP Swedish, Florida and London mutations, and the presenilin 1 (PS1) M146L and L286V mutations via Quikchange site-directed mutagenesis kit (Agilent Technologies, Inc., Santa Clara, CA, USA). All of the experimental procedures were conducted according to the manufacturer's instructions. Transgenic assays were performed according to the methods described by Devi et al (10). Normal control and APP/PS1 rats were maintained in stainless-steel cages with food and water available.

Treatment with $\mathrm{Rg} 1$. AD rats were fed with $0.5 \% \mathrm{Rg} 1$-enriched food (Langze Medicine Company, Fujian, China) for two months, prior to further analyses. All of the rats were treated in accordance with the guiding principles for the Care and Use of Animals of Fujian Medical University (Fujian, China).

Preparation of brain tissue lysates. After two months, tissue lysates were prepared from the brains of the rats. The rats were anesthetized with sodium pentobarbital $(30 \mathrm{mg} / \mathrm{kg}$; SigmaAldrich, St. Louis, MO, USA) and sacrificed, prior to isolation of brain tissue. Briefly, the brain tissue was carefully isolated and incubated with $0.25 \%$ trypsin-EDTA (Sigma-Aldrich) at $37^{\circ} \mathrm{C}$ for $1 \mathrm{~h}$. The incubated tissue was then lysed in $1 \mathrm{ml}$ sample buffer (125 mM tris-HCl, pH 6.8; 2\% SDS; 5\% glycerol; $0.003 \%$ bromophenol blue; and 1\% 2-ME; Sigma-Aldrich). The final protein concentrations of the brain tissue lysates were determined using a Bicinchoninic Acid assay (Pierce
Biotechnology, Inc., Rockford, IL, USA), according to the manufacturer's instructions. The tissue lysates were stored at $-80^{\circ} \mathrm{C}$ until required.

mRNA extraction and reverse transcription-polymerase chain reaction ( $R T-P C R)$. RNA was extracted from the brain tissue using RNAsimple Total RNA kit (TIANGEN Biotech Co., Ltd., Beijing, China). cDNA was reverse transcribed from 2 mg total mRNA using SuperScript ${ }^{\mathrm{TM}}$ III First-Strand Synthesis system (Invitrogen Life Technologies, Carlsbad, CA, USA), according to the manufacturer's instructions. The specific primers for the endoplasmic reticulum (ER) stress-associated genes, glucose-regulated protein 94 (Grp94), tumor necrosis factor receptor-associated factor 2 (TRAF2), $\mathrm{X}$-box-binding protein 1 (XBP1) and eukaryotic initiating factor (eIF) $2 \alpha$, were synthesized by Sangon Biotech Co., Ltd. (Shanghai, China) according to Table I $(11,12)$. Quantitative RT-PCR was conducted using SYBR Premix ExTaq II (Takara Biotechonology, Co., Ltd., Dalian, China), and detected with the ABI 7500 Real-Time PCR system (Applied Biosystems Life Technologies, Foster City, CA, USA). The reaction products were amplified by PCR in a volume of $50 \mu 1$, containing $1 \mu l$ forward primer, $1 \mu l$ reverse primer, $1 \mu 1$ Taq polymerase, $21 \mu \mathrm{l}$ cDNA made up to $50 \mu 1$ with water, under the following conditions: 30 cycles at $94^{\circ} \mathrm{C}$ for $30 \mathrm{sec}, 54^{\circ} \mathrm{C}$ for $30 \mathrm{sec}$ and $72^{\circ} \mathrm{C}$ for $40 \mathrm{sec}$. Following PCR, the products were electrophoresed on $1.5 \%$ agarose gel, and the gel images were digitally captured using a charge-coupled device camera (Bio-Rad Laboratories, Inc., Hercules, CA, USA), prior to analysis with the National Institutes of Health (NIH) Imager beta version 2 (NIH, Bethesda, MA, USA). The relative transcriptional value of each gene in the semi-quantitative RT-PCR experiments are presented as a ratio of the signal value, relative to that of $\beta$-actin.

Western blot analysis. The brain tissue lysates were centrifuged at $10,000 \times \mathrm{g}$ for $30 \mathrm{~min}$. Equal quantities of protein $(50 \mu \mathrm{g})$ from every group were separated by SDS-PAGE, using $15 \%$ gradient tris/glycine gels, and then transferred to polyvinylidene difluoride membranes (EMD Millipore, Billerica, MA, USA). The membranes were blocked in 5\% non-fat milk for $1 \mathrm{~h}$, and then incubated with the following primary antibodies: Mouse monoclonal anti-caspase-3 (1:2,000; cat. no. sc-7148, Santa Cruz Biotechnology, Inc., Dallas, TX, USA), mouse monoclonal anti-Grp78 (1:3,000; cat. no. sc-1050, Santa Cruz Biotechnology, Inc.), rabbit polyclonal anti-B-cell lymphoma 2 (Bcl-2)(1:3,000; cat. no. sc-492, Santa Cruz Biotechnology, Inc.), mouse monoclonal anti-Bcl-2 associated $\mathrm{X}$ protein (Bax) (1:2,000; cat. no. sc-23959, Santa Cruz Biotechnology, Inc.), mouse monoclonal anti-inositol-requiring enzyme 1 (IRE1) (1:2,000; cat. no. sc-390960, Santa Cruz Biotechnology, Inc.), mouse monoclonal anti-activating transcription factor 6 (ATF6) (1:1,000; cat. no. sc-166659, Santa Cruz Biotechnology, Inc.), rabbit polyclonal anti-protein kinase RNA-like ER kinase (PERK) (1:2,000; cat. no. ab-156919, Abcam, Cambridge, $\mathrm{UK})$, mouse monoclonal anti-cAMP response element-binding transcription factor homologous protein (CHOP) $(1: 2,000$; cat. no. sc-7351; Santa Cruz Biotechnology, Inc.), mouse monoclonal anti-caspase-12 (1:1,000, cat. no. Str-AAP-122C; Stressgen Biotechnologies Corporation, San Diego, USA) and 
Table I. Sequences of the primers used for semi-quantitative reverse transcription-polymerase chain reaction analysis, to determine the mRNA expression of endoplasmic reticulum stress-associated genes.

\begin{tabular}{|c|c|c|}
\hline Gene & Primers & Sequence \\
\hline TRAF2 & $\begin{array}{c}\text { Sense } \\
\text { Anti-sense }\end{array}$ & $\begin{array}{l}\text { 5'-AAAGGGTCAGGAAGCCGTAG-3' } \\
\text { 5'-CCGCACATAGGAATTCTTGG-3' }\end{array}$ \\
\hline Grp94 & $\begin{array}{c}\text { Sense } \\
\text { Anti-sense }\end{array}$ & $\begin{array}{l}\text { 5'-GGCCAGTTTGGTGTCGGTTT-3' } \\
\text { 5'-CTGGCCCCGTCCTAGAGTGTT-3' }\end{array}$ \\
\hline XBP1 & $\begin{array}{c}\text { Sense } \\
\text { Anti-sense }\end{array}$ & $\begin{array}{l}\text { 5'-CCTTGTAGTTGAGAACCAGG-3' } \\
\text { 5'-GGGGCTTGGTATATATGTGG-3' }\end{array}$ \\
\hline $\mathrm{eIF} 2 \alpha$ & $\begin{array}{c}\text { Sense } \\
\text { Anti-sense }\end{array}$ & $\begin{array}{l}\text { 5'-GCGAATTCATGCCGGGGCTAAGTTGTAG-3' } \\
\text { 5'-CGCTCGAGTTAATCTTCAGCTTTGGCTT-3' }\end{array}$ \\
\hline
\end{tabular}

mouse monoclonal anti- $\beta$-actin (1:3,000; cat. no. sc-8432; Santa Cruz Biotechnology, Inc.) at $4^{\circ} \mathrm{C}$ overnight. The membranes were then washed and incubated with horseradish peroxidase (HRP)-conjugated goat anti-rabbit and rabbit anti-mouse secondary antibodies (1:4,000; Santa Cruz Biotechnology, Inc.) for $2 \mathrm{~h}$ at room temperature. Immunoreactive bands were visualized using the SuperSignal West Pico Chemiluminescent substrate (Pierce Biotechnology, Inc.) and a ChemiDoc XRS system with Quantity One software (Bio-Rad Laboratories, Inc.).

Terminal deoxynucleotidyl-transferase-mediated dUTP nick end labeling (TUNEL) assay. Potential DNA fragments were examined using the TUNEL Apoptosis Detection kit (EMD Millipore, Temecula, CA, USA). Briefly, the cells were fixed with $4 \%$ paraformaldehyde in $0.1 \mathrm{M} \mathrm{NaH}_{2} \mathrm{PO}_{4}(\mathrm{pH} 7.4)$, and endogenous peroxidase was inactivated using $3 \% \mathrm{H}_{2} \mathrm{O}_{2}$. The cells were incubated with the solution containing biotin-dUTP and terminal deoxynucleotidyly transferase for $60 \mathrm{~min}$. After end-horseradish peroxidase, stained with diaminobenzidine, and counterstained with ethyl green to detect biotin-labeled nuclei. Apoptotic bodies were stained brown. The number of positively-stained cell nuclei was counted under a light microscope (CX31; Olympas, Tokyo, Japan) in three fields, by at least three independent observers.

Immunohistochemistry. The indirect streptavidin-biotin-peroxidase (SBP) complex method (SBP kits; Wuhan Boster Biological Technology, Wuhan, China) was used to visualize $4-\mu \mathrm{m}$ cryostat serial sections of the brain tissue. The cryostat serial sections were cut using a Microm HM 500 M Cryostat (Menzel-Gläser, Brunswick, Germany) and were placed on Superfrost Plus Gold glass slides (Menzel-Gläser). The sections were incubated with rabbit polyclonal antibodies against rat filamentous NFTs (1:1,000; cat. no. ab136407, Abcam) and A $\beta$ (1:2,000, cat. no. ab-120851, Abcam). Antigen-antibody binding was determined using a HRP-labeled polymer conjugated to goat anti-rabbit secondary antibodies (1:1,000, cat. no. ab-6721; Abcam), using the dextranpolymer technique (DAKO, Tokyo, Japan). The immunohistochemical images were observed and captured using a photomicroscope (CX31; Olympus, Tokyo, Japan).
Statistical analysis. Statistical analyses were performed using SPSS version 20.0 (IBM SPSS, Armonk, NY, USA). Student's two-tailed t-test was used to compare protein and mRNA expression in the brain tissue between the AD model and normal control rats. All results are expressed as the mean \pm standard deviation. In order to determine the significance of differences between groups, an analysis of variance was conducted. $\mathrm{P}<0.05$ was considered to indicate a statistically significant difference.

\section{Results}

NFTs and A $\beta$ plaques are decreased in Rg1-treated AD rats. The present study determined the effects of Rg1 treatment on the formation of $\mathrm{A} \beta$ plaques and NFTs. NFTs were significantly decreased in the Rg1-treated rats, compared with that in the $\mathrm{AD}$ rats (Fig. 1). The number of $\mathrm{A} \beta$ plaques were also significantly decreased in the Rg1 rats, compared with that in the AD rats (Fig. 1).

Rg1 inhibits cell apoptosis in AD rats. Cell apoptosis of the rat brain tissue was detected by a western blot analysis of caspase- 3 and TUNEL analysis. The protein levels of activated caspase- 3 were significantly enhanced in the $\mathrm{AD}$ rats, as compared with the control rats (Fig. 2A). Notably, the activation of caspase- 3 was significantly decreased in the Rg1-treated rats, as compared with the $\mathrm{AD}$ rats (Fig. 2A; P<0.01). Furthermore, the levels of caspase-3 in the Rg1-treated rats were similar to the levels observed in the control rats.

The TUNEL assay also demonstrated that treatment with $\operatorname{Rg} 1$ may affect apoptosis in $\mathrm{AD}$ rats. The number of TUNEL-positive cells was significantly decreased in the Rg1-treated rats, compared with that in the AD rats (Fig. 2B). These results suggest that Rg1 treatment may inhibit the apoptosis of cells in the brain tissue of $\mathrm{AD}$ rats.

Rg1 inhibits apoptosis by blocking ER stress. Grp78 has been identified as a marker of ER stress, and Bcl-2 and Bax are markers of the mitochondrial apoptotic pathway $(13,14)$. Therefore the expression of the Grp78, Bcl-2 and Bax proteins were detected by western blot analysis. The protein 
Rg1 chowed brain tissue

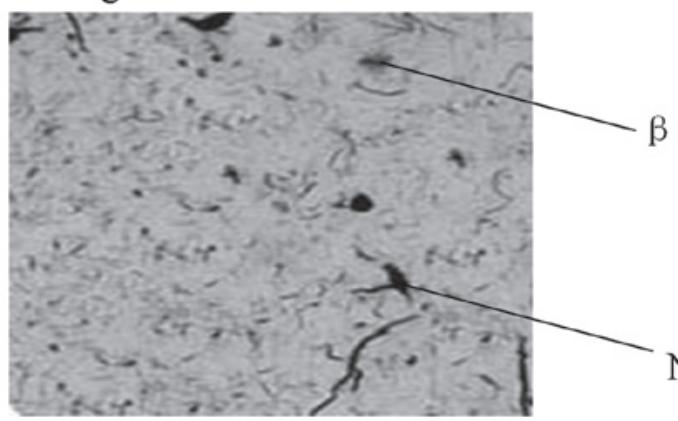

PD rats brain tissue

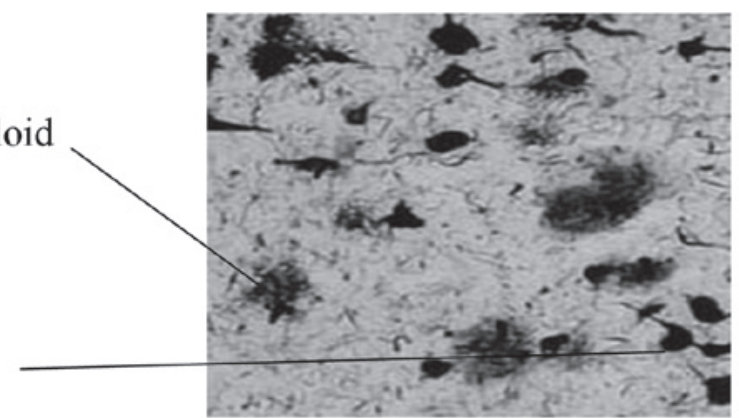

Figure 1. A $\beta$ plaques and NFTs in the brain of rats with AD. Immunostaining demonstrated the presence of more and larger A $\beta$ plaques, and NFTs in the AD rats, compared with Rg1-treated rats. $\mathrm{AD}$, Alzheimer's disease; $\mathrm{A} \beta, \beta$-amyloid; NFTs, neurofibrillary tangles.

A

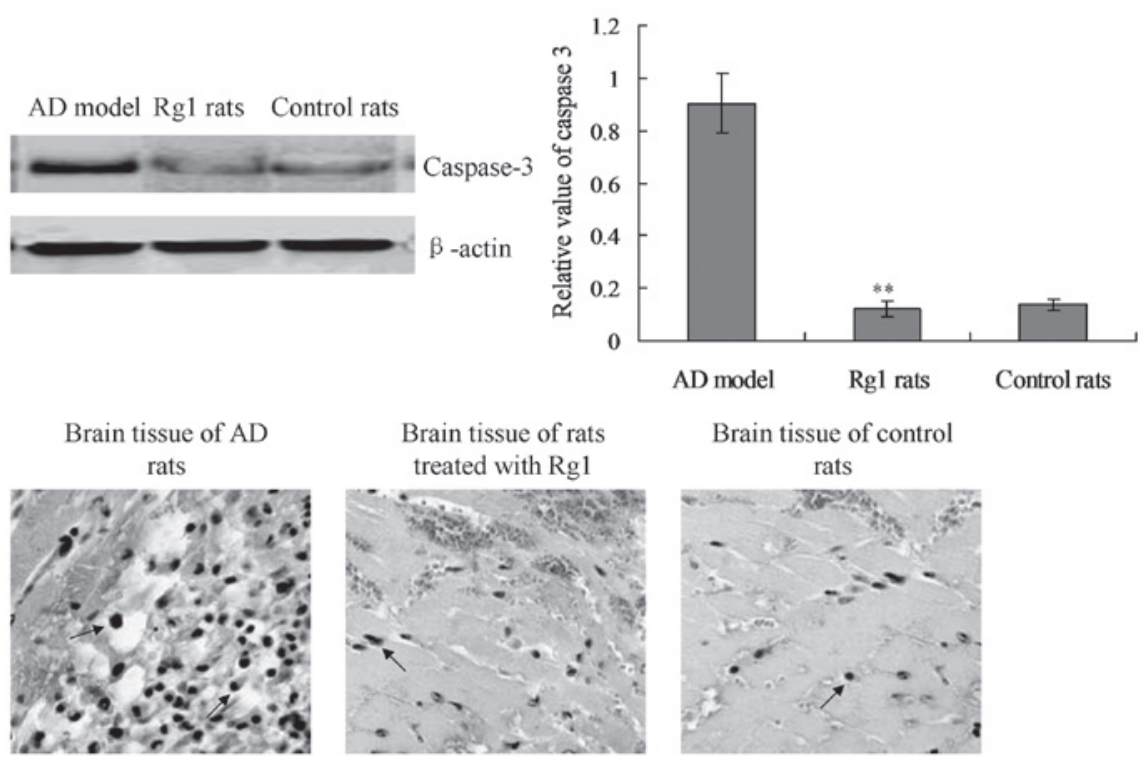

Figure 2. Rg1 inhibits apoptosis in AD rats. (A) Western blot analysis for the detection of caspase-3 protein expression levels. ${ }^{* *} \mathrm{P}<0.01$, compared with $\mathrm{AD}$ model rats. (B) Terminal deoxynucleotidyl-transferase-mediated dUTP nick end labeling assay to identify apoptotic cells. AD, Alzheimer's disease.

A

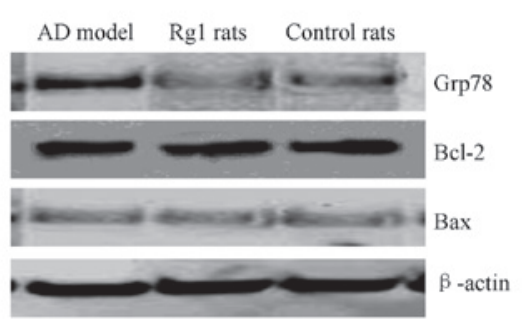

B

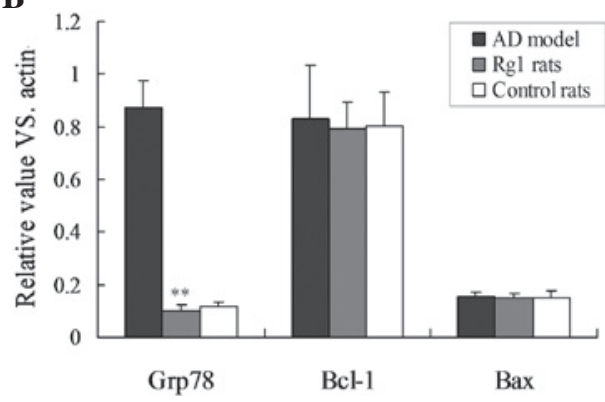

Figure 3. Alterations to the protein expression levels of Grp78, Bcl-2 and Bax in the brain tissues of AD mice. (A) Western blot analysis of Grp78, Bcl-2 and Bax. (B) Statistical analysis of western blotting. The relative expression value of each protein was calculated by comparing the gray numerical value of the protein with that of $\beta$-actin. The relative expression value of each protein was evaluated based on three independent blots, and is presented as the mean \pm standard deviation. ${ }^{* *} \mathrm{P}<0.01$, compared with the AD model rats. AD, Alzheimer's disease; Grp78, glucose-regulated protein 78; Bcl-2, B-cell lymphoma 2; Bax; Bcl-2-associated X protein.

expression levels of Grp78 were significantly lower in the Rg1-treated rats, compared with that in the AD rats (Fig. 3; $\mathrm{P}<0.01)$. However, no significant differences were detected in the protein expression levels of the mitochondria-associated apoptosis markers, Bcl-2 and Bax, between the three groups (Fig. 3). These results indicate that ER stress, but not the mitochondrial pathway, is involved in Rg1-mediated neuroprotective function. 


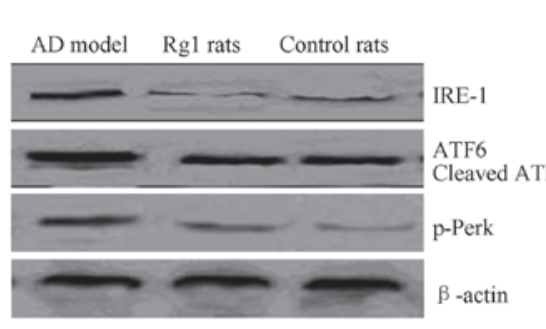

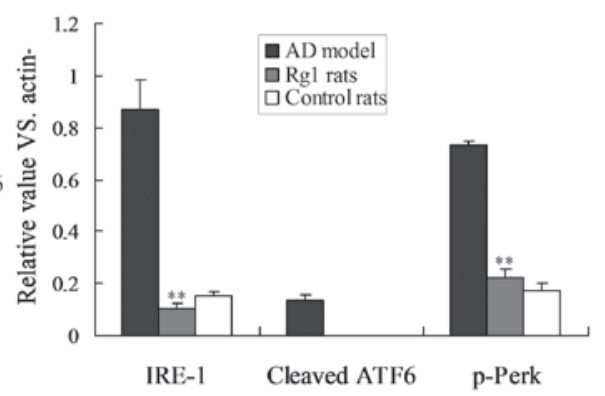

Figure 4. Determination of the expression levels of UPR pathway proteins. (A) Western blot analysis of IRE1, p-PERK and cleaved ATF6 proteins. (B) Statistical analysis of the western blotting. ${ }^{* *} \mathrm{P}<0.01$, compared with the AD model rats. AD, Alzheimer's disease; UPR, unfolded protein response; IRE-1, inositol-requiring enzyme-1; AFT6, activating transcription factor 6; p-PERK, phosphorylated protein kinase RNA-like ER kinase.

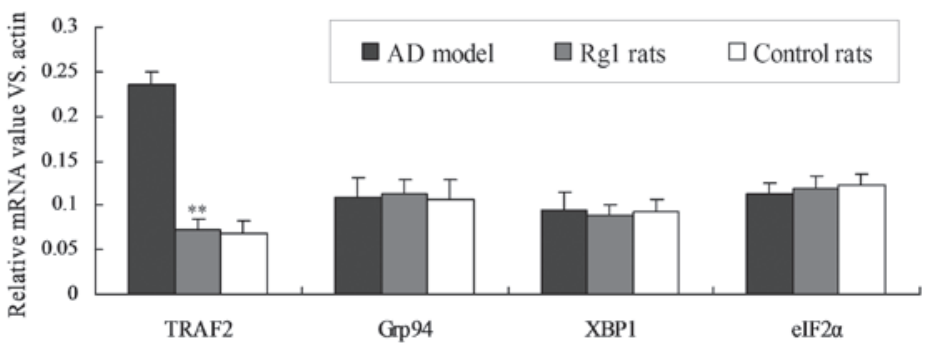

Figure 5. Semi-quantification of mRNA expression levels of the ER stress-associated genes in the brain tissue of AD rats, as determined by reverse transcription-polymerase chain reaction. The relative expression value of each gene was evaluated based on three independent blots and is presented as the mean \pm standard deviation. ${ }^{* *} \mathrm{P}<0.01$, compared with the AD model rats. AD, Alzheimer's disease; TRAF2, tumor necrosis factor receptor-associated factor 2; Grp94, glucose-regulated protein 94; XBP1, X-box-binding protein 1; eIF2 $\alpha$, eukaryotic initiating factor $2 \alpha$.

A

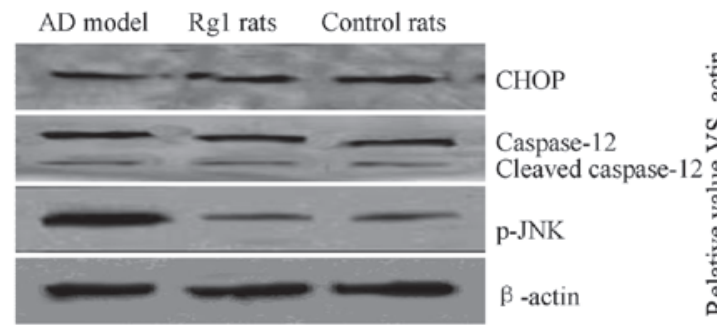

B

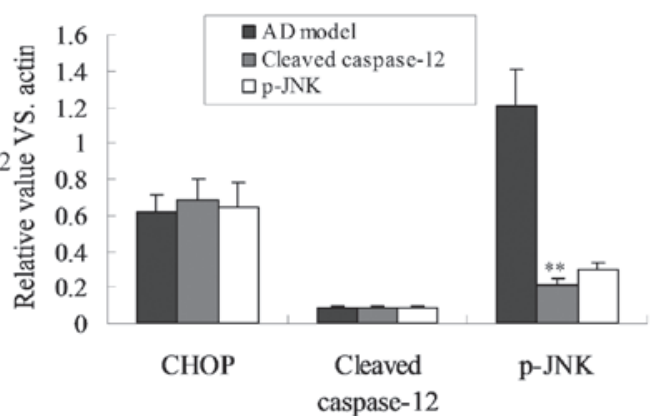

Figure 6. Changes to expression levels of ER stress-associated proteins in the brain tissue of AD rats. (A) Protein expression levels of CHOP, caspase-12 and p-JNK were evaluated by western blot analysis. (B) Statistical analysis data from western blotting. The relative expression value of each protein was evaluated based on three independent blots and is presented as the mean \pm standard deviation. ${ }^{* *} \mathrm{P}<0.01$, compared with the $\mathrm{AD}$ model rats. $\mathrm{AD}$, Alzheimer's disease; CHOP, cAMP response element-binding transcription factor homologous protein; p-JNK, phosphorylated c-Jun N-terminal protein kinase.

IRE-1 UPR pathway is involved in the inhibition of Rgl-mediated apoptosis. According to the results of the present study, Rg1 inhibits apoptosis by blocking molecules involved in ER stress. Therefore, the UPR factors involved in ER stress, including PERK, IRE-1 and ATF6, were analyzed by western blot analysis. Treatment with $\mathrm{Rg} 1$ reduced the expression of IRE-1 in the AD rats, and IRE-1 expression in the Rg1-treated rats was significantly decreased, compared with that in the AD rats (Fig. 4; P<0.01). However, p-PERK and cleaved ATF6 protein expression levels were not altered in any of the three groups (Fig. 4).
Rgl inhibits ER stress by inhibiting the transcription of ER stress-associated genes. In order to further determine the effects of Rg1 on ER stress, the transcriptional levels of numerous genes associated with ER stress, namely. TRAF2, Grp94, XBP1 and eIF2 $\alpha$, were analyzed by semi-quantitative RT-PCR (Fig. 5). The signal intensity of each PCR product was determined using a computer-assisted scanner and the relative expression value was calculated by equilibrating the signal intensity to that of $\beta$-actin. The mRNA expression of TRAF2 was significantly decreased in the Rg1-treated rats, compared with that in the $\mathrm{AD}$ rats (Fig. 5; $\mathrm{P}<0.01$ ). However, 
the expression levels of Grp94, XBP1 and eIF2 $\alpha$ was similar between the different groups. These results suggest that ER stress is inhibited in AD rats following treatment with $\mathrm{Rg} 1$.

JNK apoptotic pathway is involved in the neuroprotective effects of Rgl. In order to investigate the possible apoptotic pathways involved in the neuroprotective effects of Rg1, the JNK, CHOP and caspase-12 apoptotic pathways were evaluated by western blot analysis. The protein expression of $\mathrm{p}-\mathrm{JNK}$ was significantly increased in the AD rats, compared with that in the control rats (Fig. 6). However, when the $\mathrm{AD}$ rats were treated with $\mathrm{Rg} 1$ the p-JNK expression was shown to be significantly reduced, compared with that in the AD rats, and these expression levels were similar to those detected in the control rats (Fig. 6; $\mathrm{P}<0.01$ ). Furthermore, treatment with Rg1 had no effect on the expression of CHOP, and the cleavage of caspase-12.

\section{Discussion}

$\mathrm{A} \beta$ and intracellular NFTs are the primary pathogenic agents involved in the development of $\operatorname{AD}(3,15)$. Therefore, the formation of $\mathrm{A} \beta$ and NFTs is an important target in the treatment of AD. Furthermore, the progression of NFTs and $\mathrm{A} \beta$ accumulation is associated with the severity of the neuronal cytopathology of AD $(16,17)$. The present study aimed to explore the neuroprotective functions of $\operatorname{Rg} 1$, and to determine its underlying mechanisms, in an AD rat model. Previous studies have demonstrated that Ginsenoside Rg1 may attenuate the generation of $\mathrm{A} \beta$ and improve cognitive impairment, in a rat AD model $(17,18)$. However, the specific neuroprotective mechanisms of $\mathrm{Rg} 1$ have yet to be elucidated. Therefore, the present study aimed to investigate the possible mechanisms by which Rg1 inhibits the accumulation of NFTs and A $\beta$.

The present study demonstrated that the accumulation of NFTs and A $\beta$ was significantly decreased in AD rats, following treatment with $\mathrm{Rg} 1$. Dai et al (19) previously indicated that $A \beta$ 1-42 may induce neurotoxicity, by initiating the apoptosis of rat primary hippocampal neurons. Therefore, the present study investigated the level of cell apoptosis in the brain tissue of AD rats. Caspase- 3 levels and TUNEL-positive cells were significantly reduced in $\mathrm{Rg} 1$-treated rats, compared with those in $\mathrm{AD}$ rats, thus indicating that apoptosis was inhibited in the brain tissue of AD rats, following treatment with $\mathrm{Rg} 1$. Therefore, it is hypothesized that the accumulation of NFTs and A $\beta$ may be caused by apoptosis of brain tissue. This result is in accordance with findings from previous studies $(20,21)$.

Three primary apoptotic pathways have previously been identified: Mitochondrial, ER stress and death receptor pathways (22). It is well-known that Bcl-2 and Bax are key biomarkers for the mitochondria-mediated apoptotic pathway (11), whereas Grp78 is the critical biomarker for the ER stress-mediated apoptotic pathway (23). Therefore, the present study detected the expression levels of Bcl-2, Bax and Grp78 in the rat AD model and Rg1-treated rats. There was no significant difference between the expression levels of Bcl-2 and Bax in the AD model and Rg1-treated rats. However, the Grp78 expression levels were significantly reduced in the brain tissue of the Rg1-treated rats, compared with those in the AD rats $(\mathrm{P}<0.01)$. These results indicate that the ER stress-associated apoptotic pathway is involved in the neuroprotective function of $\mathrm{Rg} 1$.

In order to investigate the underlying mechanism of Rg1 in neuroprotection, the expression of the ER stress UPR pathway-associated proteins, p-PERK, IRE-1 and cleaved ATF6, was measured. The protein expression of IRE-1 and p-PERK was increased in the AD model rats, while it was significantly decreased in the $\mathrm{Rg} 1$-treated rats $(\mathrm{P}<0.01)$. This suggests that the PERK and IRE-1 pathway may be involved in the Rg1-mediated inhibition of the ER stress apoptotic pathway. Understanding the mechanism underlying Rg1-mediated apoptosis inhibition may help to further illuminate the role of $\mathrm{Rg} 1$ in delaying AD progression. A previous study showed that activation of IRE-1 in turn activates TRAF2 and XBP1 (24), and that PERK activation leads to phosphorylation of eIF-2 $\alpha$ (25). The expression of TRAF2, XPB1 and PERK are all associated with the subsequent initiation of apoptosis. The results of the present study provide evidence that indicates the emergence of ER stress in the AD model. Notably, treatment with Rg1 could inhibit the mRNA transcription of TRAF2 in the AD rat model. Therefore it may be hypothesized that treatment with Rg1 may inhibit the formation of NFTs and A $\beta$ in AD.

An important ER stress-mediated apoptotic pathway is the JNK pathway, which is mediated by IRE-1 (26). The IRE-1 cytoplasmic domain interacts with the adaptor protein, TRAF2, which subsequently phosphorylates and activates JNK. In the present study IRE-1 and TRAF2 expression was shown to be altered in Rg1-treated rats. Furthermore, the ER stress-associated proteins, cleaved caspase-12 and CHOP, were also measured in order to identify the specific apoptotic proteins involved in the development of AD. Xu et al (27) previously reported that cleaved caspase-12 activates caspase-3 and triggers apoptosis, and that $\mathrm{CHOP}$ and p-JNK directly induce ER stress-associated apoptosis. The present study demonstrated that there was no significant changes to the expression of $\mathrm{CHOP}$ and cleaved caspase-12 in any of the three of groups $(\mathrm{P}>0.05)$. Notably, when treated with Rg1 the p-JNK expression in AD rats was significantly decreased, compared with that in the AD model rats $(\mathrm{P}<0.05)$. These results suggest that treatment with Rg1 may inhibit p-JNK pathway-induced apoptosis, and indirectly inhibit the accumulation of NFTs and A $\beta$. Thus, Rg1 may block p-JNK-induced ER stress, and slow the progression of $\mathrm{AD}$.

In conclusion, in the present study Rg1 was shown to act as an important factor that inhibits the accumulation of NFTs and $\mathrm{A} \beta$, through blocking the ER stress-mediated apoptotic pathway. Blocking of this pathway was triggered by the IRE-1 and TRAF2 pathway, via inhibition of the expression of p-JNK.

\section{References}

1. Xing S, Shen D, Chen C, Wang J, Liu T and Yu Z: Regulation of neuronal toxicity of $\beta$-amyloid oligomers by surface ATP synthase. Mol Med Rep 8: 1689-1694, 2013.

2. He Y,Zhao H and Su G: Ginsenoside Rg1 decreases neurofibrillary tangles accumulation in retina by regulating activities of neprilysin and PKA in retinal cells of AD rats model. J Mol Neurosci 52: 101-106, 2014.

3. Galimberti D and Scarpini E: Progress in Alzheimer's disease. J Neurol 259: 201-211, 2012.

4. Sima X, Xu J, Li J, Zhong W and You C: Expression of $\beta$-amyloid precursor protein in refractory epilepsy. Mol Med Rep 9: 1242-1248, 2014. 
5. Sethi KD: Clinical aspects of Parkinson disease. Curr Opin Neurol 15: 457-460, 2002

6. Goes AT, Souza LC, Filho CB, Del Fabbro L, De Gomes MG, Boeira SP and Jesse CR: Neuroprotective effects of swimming training in a mouse model of Parkinson's disease induced by 6-hydroxydopamine. Neuroscience 256: 61-71, 2014.

7. Wu J, Pan Z, Cheng M, Shen Y, Yu H, Wang Q and Lou Y: Ginsenoside Rg1 facilitates neural differentiation of mouse embryonic stem cells via GR-dependent signaling pathway. Neurochem Int 62: 92-102, 2013.

8. Zhuang P, Zhang Y and Pang T: Proliferation effect of neural stem cell of ginsenoside Rg1 in vitro. Zhongguo Zhong Yao Za Zhi 34: 443-446, 2009 (In Chinese).

9. Lu MC, Lai TY, Hwang JM, Chen HT, Chang SH, Tsai FJ, Wang HL, Lin CC, Kuo WW and Huang CY: Proliferationand migration-enhancing effects of ginseng and ginsenoside Rg1 through IGF-1 and FGF-2 signaling pathways on RSC96 Schwann cells. Cell Biochem Funct 27: 186-192, 2009.

10. Devi L and Ohno M: Genetic reductions of beta-site amyloid precursor protein-cleaving enzyme 1 and amyloid-beta ameliorate impairment of conditioned taste aversion memory in 5XFAD Alzheimer's disease model rats. Eur J Neurosci 31: $110-118,2010$

11. Xu K, Wang X, Shi Q, Chen C, Tian C, Li XL, Zhou RM, Chu YL and Dong XP: Human prion protein mutants with deleted and inserted octarepeats undergo different pathways to trigger cell apoptosis. J Mol Neurosci 43: 225-234, 2011.

12. Lee SK and Kim YS: Phosphorylation of eIF2 $\alpha$ attenuates statin-induced apoptosis by inhibiting the stabilization and translocation of p53 to the mitochondria. Int J Oncol 42 : 810-816, 2013.

13. Hegde RS, Mastrianni JA, Scott MR, DeFea KA, Tremblay P, Torchia M, DeArmond SJ, Prusiner SB and Lingappa VR: A transmembrane form of the prion protein in neurodegenerative disease. Science 279: 827-834, 1998.

14. Li Z, Shen J, Chen Y, Pan J, Zeng H, Fang H, Ye Z, Zeng C, Zhang $\mathrm{R}$ and Cai D: Mitochondrial genome sequenceing of chondrocytes in osteoarthritis by human mitochondria RT2 Profiler $^{\text {TM }}$ PCR array. Mol Med Rep 6: 39-44, 2012.

15. Zhao H, Chang R, Che H, Wang J, Yang L, Fang W, Xia Y, Li N, Ma Q and Wang X: Hyperphosphorylation of tau protein by calpain regulation in retina of Alzheimer's disease transgenic mouse. Neurosci Lett 551: 12-16, 2013.
16. Lai MK, Chen CP, Hope T and Esiri MM: Hippocampal neurofibillary tangle changes and aggressive behaviour in dementia. Neuroreport 21: 1111-1115, 2010.

17. Kril JJ, Patel S, Harding AJ and Halliday GM: Neuron loss from the hippocampus of Alzheimer's disease exceeds extracellular neurofibrillary tangle formation. Acta Neuropathol 103: 370-376, 2002.

18. Chen LM, Lin ZY, Zhu YG, Lin N, Zhang J, Pan XD and Chen XC: Ginsenoside $\operatorname{Rg} 1$ attenuates $\beta$-amyloid generation via suppressing PPAR $\gamma$-regulated BACE1 activity in N2a-APP695 cells. Eur J Pharmacol 675: 15-21, 2012.

19. Dai X, Chang P, Zhu Q, Liu W, Sun Y, Zhu S and Jiang Z: Chitosan oligosaccharides protect rat primary hippocampal neurons from oligomeric $\beta$-amyloid 1-42-induced neurotoxicity. Neurosci Lett 554: 64-69, 2013.

20. Chen Z and Zhong C: Decoding Alzheimer's disease from perturbed cerebral glucose metabolism: implications for diagnostic and therapeutic strategies. Prog Neurobiol 108: 21-43, 2013.

21. Salminen A, Kaarniranta K, Kauppinen A, Ojala J, Haapasalo A, Soininen H and Hiltunen M: Impaired autophagy and APP processing in Alzheimer's disease: The potential role of Beclin 1 interactome. Prog Neurobiol 106-107: 33-54, 2013.

22. Gillies LA and Kuwana T: Apoptosis regulation at the mitochondrial outer membrane. J Cell Biochem 115: 632-640, 2014

23. Wang X, Shi Q, Xu K, Gao C, Chen C, Li XL, Wang GR, Tian C, Han J and Dong XP: Familial CJD associated PrP mutants within transmembrane region induced Ctm-PrP retention in ER and triggered apoptosis by ER stress in SH-SY5Y cells. PLoS One 6: e14602, 2011.

24. Fan Q, Hu Y, Pang H, Sun J, Wang Z and Li JM: Melittin protein inhibits the proliferation of MG63 cells by activating inositol-requiring protein- $1 \alpha$ and X-box binding protein 1-mediated apoptosis. Mol Med Rep 9: 1365-1370, 2014.

25. Kimball SR and Jefferson LS: Induction of REDD1 gene expression in the liver in response to endoplasmic reticulum stress is mediated through a PERK, eIF2 $\alpha$ phosphorylation, ATF4-dependent cascade. Biochem Biophys Res Commun 427: 485-489, 2012.

26. Yenki P, Khodagholi F and Shaerzadeh F: Inhibition of phosphorylation of JNK suppresses A $\beta$-induced ER stress and upregulates prosurvival mitochondrial proteins in rat hippocampus. J Mol Neurosci 49: 262-269, 2013.

27. Xu K and Zhu XP: Endoplasmic reticulum stress and prion diseases. Rev Neurosci 23: 79-84, 2012. 